\title{
On the behaviour of polymer insulator with deposited moss on the surface against lightning in polluted air condition
}

\begin{abstract}
Deposited moss on the surface of polymer insulators can be effective on the electrical performance of insulator and network as well. This problem can be sensed especially in the forest areas and tropical countries. In the tropical countries like Malaysia beside of local factors for creation of moss on the surface of insulator, the air pollution plays an important role as well. In this study a $10 \mathrm{kV}$ polymer insulator has been chosen an the electrical performance of insulator against lightning was considered through both simulation and experimental works and the results have be discussed accordingly. The results show that the contaminated insulator have different behaviour against lightning and in order to reach a proper insulation level for line, the local factors should be taken into account.
\end{abstract}

Keyword: Polymer insulator; Moss; Electric field; Lightning 